\title{
Design of a Flexible Force-Sensing Platform for Medical Ultrasound Probes
}

Conference Paper · June 2016

DOI: 10.1109/BIOROB.2016.7523638

CITATIONS

9 authors, including:

Adrian Schoisengeier

King's College London

3 PUBLICATIONS 44 CITATIONS

SEE PROFILE

Junghwan Back

20 PUBLICATIONS 112 CITATIONS

SEE PROFILE

Some of the authors of this publication are also working on these related projects:

Project Development of a Robotic Trans-esophageal Ultrasound System View project

Project Multimodality Medical Image Fusion View project
READS

242

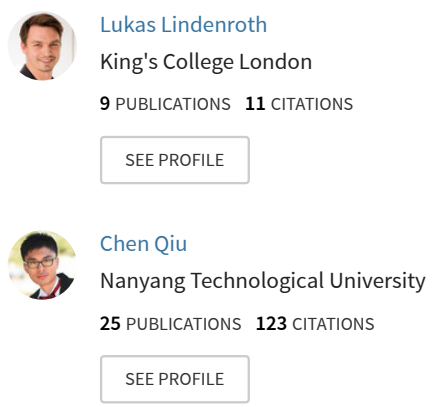




\title{
Design of a Flexible Force-Sensing Platform for Medical Ultrasound Probes
}

\author{
Adrian Schoisengeier, Lukas Lindenroth, Junghwan Back, Chen Qiu, Yohan Noh, Kaspar Althoefer, \\ Jian S Dai, Kawal Rhode, Hongbin Liu
}

\begin{abstract}
Automated ultrasound scanning is a growing research field. However, existing platforms for mounting the ultrasound probe do not possess any soft, compliant properties that would ensure the safety of the patient. Moreover, many current ultrasound manipulators do not include tactile feedback or employ rather expensive commercial force sensors. This paper proposes the design of a flexible platform with soft joints. The device equips an ultrasound manipulator with both compliant behaviour and 6-axis force feedback without the need of a commercial force sensor. A general methodology was developed to derive the symbolic compliance matrix of such a flexible mechanism. Subsequently, a finite element analysis of the platform was carried out and the results were compared to the analytical solutions. The results show that force sensing based on the analytical method has an error of 5-16\% compared to the FEA simulation, depending on the degree of freedom.
\end{abstract}

\section{INTRODUCTION}

Ultrasound diagnosis is a non-invasive medical procedure that involves less risks and less dexterity than other surgical techniques [1]. Additionally, ultrasound examinations are more cost-effective and portable than other non-invasive diagnosis methods, such as CT (Computer Tomography) and MRI (Magnetic Resonance Imaging). This makes ultrasonography particularly suitable for being carried out by a machine. Robotic ultrasound probe manipulators that perform medical tasks can have a variety of promising applications. In the past decade, several systems have been developed, providing solutions for kidney examinations, the detection of internal bleedings after accidents [2], and several other functions [3,4]. An emerging application is teleechography $[2,5,6]$, where a robotic manipulator is remotely controlled by a distant sonographer who cannot reach the patient because of time constraints or geographical reasons. Moreover, automated ultrasound scanners can be a solution to the prevalent fatigue issues of professional sonographers [7].

This paper proposes a flexible platform that equips a robotic ultrasound manipulator with both a compliant behaviour and 6-axis force feedback, without the need of a commercial force sensor. The implementation of soft, flexible structures in robotic systems is particularly useful for medical applications. It can ensure the safety of the patient and allows a gentler interaction with the human body. In the worst-case scenario of a system malfunction, soft materials, such as silicone or rubber, are more likely to break rather than

A. Schoisengeier, L. Lindenroth, J. Back, C. Qiu, Y. Noh, K. Althoefer, J.S. Dai, H. Liu are with the Centre for Robotics Research, Department of Informatics, King's College London. K. Rhode is with the Biomedical Engineering Department, King's College London. (corresponding author: Hongbin Liu, e-mail: hongbin.liu@kcl.ac.uk) harming the patient. As the ultrasound manipulator interacts with the dynamic environment of a human body, tactile feedback is indispensable for the proper control of such an instrument. A commercial force sensor can be employed for this task, but such device increases the overall cost of the system. It would be more cost-effective to deduce the forces from displacement measurements, as displacement sensors are generally less expensive than accurate force sensors. In fact, when using soft materials, the manipulator will undergo deformations that can be detected with such sensors Furthermore, displacement sensors do not have to be placed inside the manipulator, but can perform the measurements externally.

A variety of robotic ultrasound probe manipulators has been developed in the past decade. Mustafa et al. presented in [8] a design that uses an industrial 6-axis robot to manipulate an ultrasound probe and mimics the movements of a professional sonographer. A conventional 6-axis force sensor was employed in order to apply a constant force to the body of the patient. A more compact prototype was presented in [9] by Sen et al. The device uses a conventional force sensor, placed between the end-effector and the ultrasound probe. However, compliant elements are not present in both of these systems. Similarly, most of the other examined ultrasound probe manipulators (i.e. [10]-[12]) use commercial force/torque sensors for tactile feedback and do not employ soft materials. A device that uses elastic components is the system presented by Ito in [13], where mechanical springs act as compliant elements in order to maintain a constant contact force to the body. However, there is no force feedback integrated in this device. Nakadate et al. presented in [14] a customised force sensing system for ultrasound probes that is based on displacement measurements. It employs elastic materials (i.e. sponge), which are placed between the probe and the outer probe case. Jentoft et al. developed in [15] an angle sensor for compliant joints, based on optical fibres placed inside the soft material. Their system demonstrates the feasibility of displacement measurements of soft materials that do not have a fixed centre of rotation. Another method to perform force sensing from displacement measurements involves ortho-planar flexure mechanisms, as presented in [16]-[18]. Even though the size of these elements is in the order of a couple of centimetres, the working principle can be applied to a larger scale as well.

\section{PLATFORM DESIGN}

The proposed design for the ultrasound probe platform is shown in Figure 1. It comprises two rigid components (green colour), and three flexible joints (blue colour). The rigid plate in the centre contains a cavity that is supposed to hold the ultrasound probe. It is connected via the flexible joints to the 
outer frame. The outer frame is supposed to be attached to the end-effector of a robotic manipulator. Thus, in terms of the end-effector coordinates, the outer frame is fixed, whereas the inner probe holder is flexible. The latter will perform movements when exposed to external forces, due to the three flexible joints. As demonstrated in [16], the radial symmetry of such a ortho-planar structure is very practical for force measurements, as it results in a diagonal compliance matrix. Moreover, the planar design of this structure facilitates the installation on the end-effector of a robotic manipulator. In practise, the inner probe holder can be made of two parts, which facilitates mounting the ultrasound probe firmly.

The platform design must be suitable for the use with conventional ultrasound probes. Thus, the device should be compact and have roughly the same size as the ultrasound probe itself. Concerning compliance properties, the absolute displacements of the platform should be large enough to be measurable by typical displacement sensors for this application. In opposition to this, the platform's displacements should be limited to less than $10 \%$ of the beam length. This ensures that linear approximations of the beam deflections are still valid. Therefore, an optimal trade-off between these two constraints for the beam properties has to be found, while keeping the overall size of the platform as compact as possible.

The dimensions of the platform in Figure 1 are $L=$ $40 \mathrm{~mm}, h=15 \mathrm{~mm}, b=5 \mathrm{~mm}$ for beam length, height, width respectively, and $r=40 \mathrm{~mm}$ for the radius of the inner probe holder. The urethane rubber PMC-780, with a Young's modulus of approximately $E=9 \mathrm{MPa}$ and a shear modulus of $G=3 \mathrm{MPa}$, was selected as reference material for the flexible joints. Given these parameters, the platform obtains satisfying compliance properties. This was tested by inserting the values for $E, G, L, h, b, r$ into the compliance matrix of the platform, derived in SECTION IV, equations (25).

\section{Compliance Analysis of The FleXible Joints}

As illustrated in Figure 1, the platform structure is composed of three flexible segments, which make the inner part of the platform compliant to external forces. In order to predict the displacement of the platform when subjected to forces, it is first necessary to determine the elastic properties of each flexible segment separately. For this purpose, the three-dimensional compliance matrix of such a flexible segment will be formulated, using two different approaches: the Euler-Bernoulli Beam Theory, and the Timoshenko Beam Theory. Given the working principle of the proposed device, all forces will act on the inner part of platform, whereas the outer part is considered fixed. Therefore, each of the three flexible joints can be modelled as a cantilever beam. Figure 2 illustrates the case of a cantilever beam with a load and torque acting on its free end.

The three flexible segments of the platform will be considered as homogenous beam elements with a Young's modulus $E$ and a shear modulus $G$. Moreover, the crosssection $A=h b$ is assumed rectangular, where $h$ is the beam height, measured along the $y$-axis, and $b$ is the beam width, measured along the $z$-axis. The area moments of inertia for rotations about the $y$-axis and $z$-axis are defined as $I_{y}=\frac{1}{12} b^{3} h \quad$ and $I_{z}=\frac{1}{12} h^{3} b \quad$ respectively. Furthermore,

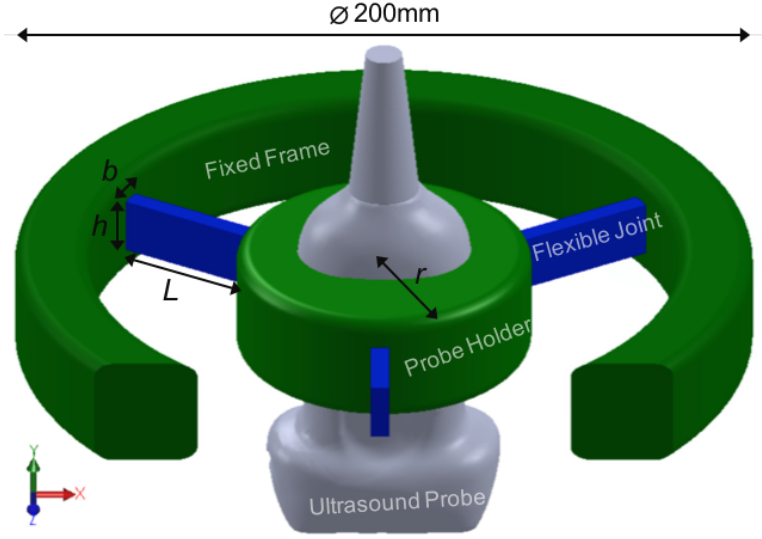

Figure 1. Section view of platform including an ultrasound probe

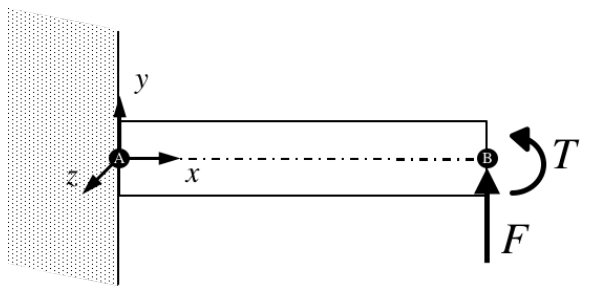

Figure 2. Illustration of a cantilever beam

assuming $h>b$, the torsion constant $J$ of the beam can be approximated as:

$$
J \approx h b^{3}\left(\frac{1}{3}-0.21 \frac{b}{h}\left(1-\frac{b^{4}}{12 h^{4}}\right)\right) .
$$

Moreover, we can introduce a 3D compliance matrix, denoted as $\boldsymbol{C}$, and a 3D stiffness matrix, denoted as $\boldsymbol{K}$, which describe the relation between an applied wrench $\boldsymbol{F}=\left(\begin{array}{llllll}F_{x} & F_{y} & F_{z} & T_{x} & T_{y} & T_{Z}\end{array}\right)^{T}$ and the resultant displacement $\boldsymbol{\Delta} \boldsymbol{X}=\left(\begin{array}{llllll}\delta_{x} & \delta_{y} & \delta_{z} & \theta_{x} & \theta_{y} & \theta_{z}\end{array}\right)^{T}$ of the free beam end, such that

$$
\begin{aligned}
& \Delta X=C \cdot F, \\
& F=K \cdot \Delta X .
\end{aligned}
$$

\section{A. Euler-Bernoulli Beam Theory}

The Euler-Bernoulli beam theory is a very common tool in engineering for calculating the deflection of a beam. It provides accurate results for slender beams under a static load, when the scale of the deflections is relatively small. Derivations of the Euler-Bernoulli beam theory for common beam configurations can be found in most university-level engineering books about structure mechanics and [19], [20]. For this specific case of a cantilever beam, all forces and torques act at the free end of the beam and can be combined to a resulting load $F$ and torque $T$. The deflection of the beam-end $\delta_{y}$ along the $y$-axis and its angle about the $z$-axis $\theta_{z}$ can be expressed as follows; using a right handed coordinate system and positive rotations acting in counterclockwise direction, as shown in Figure 2.

$$
\delta_{y}=\frac{L^{3}}{3 E I_{z}} F_{y}+\frac{L^{2}}{2 E I_{z}} T_{z}, \quad \theta_{z}=\frac{L^{2}}{2 E I_{z}} F_{y}+\frac{L}{E I_{z}} T_{z} .
$$

The relations for displacements along the $z$-axis and rotations about the $y$-axis are equivalent: 


$$
\delta_{z}=\frac{L^{3}}{3 E I_{y}} F_{z}-\frac{L^{2}}{2 E I_{y}} T_{y}, \theta_{y}=-\frac{L^{2}}{2 E I_{y}} F_{z}+\frac{L}{E I_{y}} T_{y} .
$$

The Euler-Bernoulli beam theory therefore provides four of the six required equations to create a $3 \mathrm{D}$ compliance matrix of the free end of a cantilever beam. The remaining two equations describe linear and angular deformations along the $x$-axis, i.e. the axis of the beam. One equation can be directly obtained from the definition of the Young's modulus $E$, which expresses how much a material extends under tensions or shortens under compression:

$$
E \equiv \frac{L}{\Delta L} \frac{F}{A}=\frac{L}{\delta_{x}} \frac{F_{x}}{A} .
$$

The second equation can be obtained from the definition of the shear modulus $G$, and describes how much the material twists, when subjected to torque around the $x$-axis.

$$
G \equiv \frac{L}{\theta} \frac{T}{J}=\frac{L}{\theta_{x}} \frac{T_{x}}{J} .
$$

Equations (4)-(7) can be put into matrix form by defining a $6 \times 6$ compliance matrix, henceforth denoted as $\boldsymbol{C}_{E B}$, which transforms a wrench vector $\boldsymbol{F}$ into a displacement vector $\boldsymbol{\Delta} \boldsymbol{X}$, such that

$$
\begin{gathered}
\Delta \boldsymbol{X}= \\
{\left[\begin{array}{c}
\delta_{x} \\
\delta_{y} \\
\delta_{z} \\
\theta_{x} \\
\theta_{y} \\
\theta_{z}
\end{array}\right]=\left[\begin{array}{cccccc}
\frac{L}{A E} & 0 & 0 & 0 & 0 & 0 \\
0 & \frac{L^{3}}{3 E I_{z}} & 0 & 0 & 0 & \frac{L^{2}}{2 E I_{z}} \\
0 & 0 & \frac{L^{3}}{3 E I_{y}} & 0 & -\frac{L^{2}}{2 E I_{y}} & 0 \\
0 & 0 & 0 & \frac{L}{G J} & 0 & 0 \\
0 & 0 & -\frac{L^{2}}{2 E I_{y}} & 0 & \frac{L}{E I_{y}} & 0 \\
F_{y} \\
\frac{L^{2}}{2 E I_{z}} \\
0 & 0 & 0 & \frac{L}{E I_{z}}
\end{array}\right] \cdot\left[\begin{array}{c}
F_{x} \\
F_{z} \\
T_{x} \\
T_{y} \\
T_{z}
\end{array}\right] .}
\end{gathered}
$$

On the other hand, solving the same system of six equations for the variables $F_{x}, F_{y}, F_{z}, T_{x}, T_{y}, T_{z}$ leads to the stiffness matrix $\boldsymbol{K}_{E B}=\left(\boldsymbol{C}_{E B}\right)^{-1}$, which satisfies the relation

$$
\left.\begin{array}{c}
\boldsymbol{F} \\
F_{y} \\
F_{z} \\
T_{x} \\
T_{y} \\
T_{z}
\end{array}\right]=\left[\begin{array}{cccccc}
\frac{A E}{L} & 0 & 0 & 0 & 0 & 0 \\
0 & \frac{12 E I_{z}}{L^{3}} & 0 & 0 & 0 & -\frac{6 E I_{z}}{L^{2}} \\
0 & 0 & \frac{12 E I_{y}}{L^{3}} & 0 & \frac{6 E I_{y}}{L^{2}} & 0 \\
0 & 0 & 0 & \frac{G J}{L} & 0 & 0 \\
0 & 0 & \frac{6 E I_{y}}{L^{2}} & 0 & \frac{4 E I_{y}}{L} & 0 \\
0 & -\frac{6 E I_{z}}{L^{2}} & 0 & 0 & 0 & \frac{4 E I_{z}}{L}
\end{array}\right] \cdot\left[\begin{array}{c}
\delta_{x} \\
\delta_{y} \\
\delta_{z} \\
\theta_{x} \\
\theta_{y} \\
\theta_{z}
\end{array}\right] .
$$

\section{B. Timoshenko Beam Theory}

The Timoshenko beam theory is a more accurate model to describe the deflection of beams than the Euler-Bernoulli theory. A main drawback of the latter is that it underestimates the deflection of short beams. The Timoshenko model overcomes this problem by taking into account rotational inertia effects and shear deformation. This results in a lower stiffness than predicted by the Euler-Bernoulli theory. For long beams with a slenderness ratio (length-to-depth ratio) of more than 5, the Timoshenko approach converges to the solution of the Euler-Bernoulli theory [19].

Timoshenko introduced an additional correction term denoted $\Phi$, which leads to a modified form of equation (4):

$$
\delta_{y}=\frac{\left(4+\Phi_{z}\right) L^{3}}{12 E I} F_{y}+\frac{L^{2}}{2 E I} T_{z} .
$$

The term $\Phi$ represents the effect of shear deformation on the bending of the beam. Depending on the observed axis, it is defined as [20]

$$
\Phi_{\mathrm{z}}=\frac{12 E I_{z}}{\kappa A G L^{2}}, \Phi_{\mathrm{y}}=\frac{12 E I_{y}}{\kappa A G L^{2}},
$$

where $\kappa$ is the so called Timoshenko coefficient, which is equal to $5 / 6$ for beams with rectangular cross-sections. As $\Phi$ is strictly positive, it can be concluded that beam deflections caused by forces are generally larger when using the Timoshenko model. The effect of torques, on the other hand, remains unchanged compared to the Euler-Bernoulli theory. The resulting compliance matrix $\boldsymbol{C}_{\text {TIM }}$ is defined as

$$
\begin{gathered}
\boldsymbol{\Delta} \boldsymbol{X}= \\
{\left[\begin{array}{c}
\delta_{x} \\
\delta_{y} \\
\delta_{z} \\
\theta_{x} \\
\theta_{y} \\
\theta_{z}
\end{array}\right]=\left[\begin{array}{cccccc}
\frac{L}{A E} & 0 & 0 & 0 & 0 & 0 \\
0 & \frac{\left(4+\Phi_{z}\right) L^{3}}{12 E I_{z}} & 0 & 0 & 0 & \frac{L^{2}}{2 E I_{z}} \\
0 & 0 & \frac{\left(4+\Phi_{y}\right) L^{3}}{12 E I_{y}} & 0 & -\frac{L^{2}}{2 E I_{y}} & 0 \\
0 & 0 & 0 & \frac{L}{G J} & 0 & 0 \\
0 & 0 & -\frac{L^{2}}{2 E I_{y}} & 0 & \frac{L}{E I_{y}} & 0 \\
0 & \frac{L^{2}}{2 E I_{z}} & 0 & 0 & 0 & \frac{L}{E I_{z}}
\end{array}\right] \cdot\left[\begin{array}{c}
F_{x} \\
F_{y} \\
F_{z} \\
T_{x} \\
T_{y} \\
T_{z}
\end{array}\right] .}
\end{gathered}
$$

Note that the terms $\Phi_{y}$ and $\Phi_{z}$ converge to zero when shear effects are negligible, resulting in $\boldsymbol{C}_{T I M}=\boldsymbol{C}_{E B}$. The inverse of this matrix gives the corresponding stiffness matrix of the Timoshenko beam element $\boldsymbol{K}_{T I M}$, as shown below,

$$
\begin{gathered}
\boldsymbol{F}= \\
{\left[\begin{array}{c}
F_{x} \\
F_{y} \\
F_{z} \\
T_{x} \\
T_{y} \\
T_{z}
\end{array}\right]=\left[\begin{array}{cccccc}
\frac{A E}{L} & 0 & 0 & 0 & 0 & 0 \\
0 & \frac{12}{1+\Phi_{z}} \frac{E I_{z}}{L^{3}} & 0 & 0 & 0 & \frac{-6}{1+\Phi_{z}} \frac{E I_{z}}{L^{2}} \\
0 & 0 & \frac{12}{1+\Phi_{y}} \frac{E I_{y}}{L^{3}} & 0 & \frac{6}{1+\Phi_{y}} \frac{E I_{y}}{L^{2}} & 0 \\
0 & 0 & 0 & \frac{G J}{L} & 0 & 0 \\
0 & 0 & \frac{6}{1+\Phi_{y}} \frac{E I_{y}}{L^{2}} & 0 & \frac{4+\Phi_{y}}{1+\Phi_{y}} \frac{E I_{y}}{L} & 0 \\
0 & \frac{-6}{1+\Phi_{z}} \frac{E I_{z}}{L^{2}} & 0 & 0 & 0 & \frac{4+\Phi_{z}}{1+\Phi_{z}} \frac{E I_{z}}{L}
\end{array}\right] \cdot\left[\begin{array}{c}
\delta_{x} \\
\delta_{y} \\
\delta_{z} \\
\theta_{x} \\
\theta_{y} \\
\theta_{z}
\end{array}\right] .}
\end{gathered}
$$

Detailed derivations of the $3 \mathrm{D}$ compliance and stiffness matrices based on the Timoshenko theory can be found in [19], [20].

\section{COMPliance Analysis of the EnTiRe PlatForm}

After having formulated the compliance matrix of the flexible joints in SECTION III, it is now possible to 
formulate the $3 \mathrm{D}$ compliance matrix of the entire platform. Given the stiffness matrix $\boldsymbol{K}_{\text {beam }}$ of a flexible beam element, the problem consists in constructing the overall stiffness matrix $\boldsymbol{K}_{\text {plat }}$ of a structure that is composed of a parallel chain of such beam elements. Moreover, the aim is to express this stiffness matrix with respect to the global reference frame located at the centre of the platform structure.

The so called wrench transformation matrix Ad maps a wrench $\boldsymbol{F}=\left(\begin{array}{lllllll}F_{x} & F_{y} & F_{z} & T_{x} & T_{y} & T_{z}\end{array}\right)^{T}$ from one coordinate frame to another. More specifically, a wrench $\boldsymbol{F}_{\mathcal{A}}$ expressed with respect to coordinate frame $\mathcal{A}$ can be converted to a wrench $\boldsymbol{F}_{\mathcal{B}}$ with respect to coordinate frame $\mathcal{B}$ by using the wrench transformation matrix Ad as follows [21],

$$
\boldsymbol{F}_{\mathcal{B}}=\operatorname{Ad} \boldsymbol{F}_{\mathcal{A}}, \quad \boldsymbol{F}_{\mathcal{A}}=\operatorname{Ad}^{-1} \boldsymbol{F}_{\mathcal{B}}
$$

The wrench transformation matrix can also be used to transform a displacement vector $\boldsymbol{\Delta} \boldsymbol{X}=$ $\left(\begin{array}{llllll}\delta_{x} & \delta_{y} & \delta_{z} & \theta_{x} & \theta_{y} & \theta_{z}\end{array}\right)^{T}$, using the relations

$$
\boldsymbol{\Delta} \boldsymbol{X}_{\mathcal{B}}=\mathbf{A d}^{-\mathrm{T}} \boldsymbol{\Delta} \boldsymbol{X}_{\mathcal{A}}, \quad \boldsymbol{\Delta} \boldsymbol{X}_{\mathcal{A}}=\mathbf{A d}^{\mathrm{T}} \boldsymbol{\Delta} \boldsymbol{X}_{\mathcal{B}} .
$$

If frame $\mathcal{A}$ can be obtained by performing a translation $\boldsymbol{p}=$ $\left(p_{x}, p_{y}, p_{z}\right)^{T}$ of frame $\mathcal{B}$, followed by a rotation $\boldsymbol{R}$, then

$$
\operatorname{Ad}=\left[\begin{array}{cc}
R & 0 \\
D R & R
\end{array}\right]
$$

where $\boldsymbol{D}$ is the skew-symmetric matrix that represents the cross product operation with the vector $\boldsymbol{p},[16]$, [22]

$$
\boldsymbol{D}=\left[\begin{array}{ccc}
0 & -p_{z} & p_{y} \\
p_{z} & 0 & -p_{x} \\
-p_{y} & p_{x} & 0
\end{array}\right]
$$

The transformation matrix Ad can also be used to convert a stiffness matrix $\boldsymbol{K}_{\mathcal{B}}$ in frame $\mathcal{B}$ to its equivalent $\boldsymbol{K}_{\mathcal{A}}$ in frame $\mathcal{A}$. The relation between these two matrices can be established via equations (14) and (15),

$$
\begin{aligned}
& \boldsymbol{K}_{\mathcal{A}} \boldsymbol{\Delta} \boldsymbol{X}_{\mathcal{A}} \\
& =\left[\boldsymbol{F}_{\mathcal{A}}=\mathbf{A d}^{-1} \boldsymbol{F}_{\mathcal{B}}=\mathbf{A d}^{-1}\left(\boldsymbol{K}_{\mathcal{B}} \boldsymbol{\Delta} \boldsymbol{X}_{\mathcal{B}}\right)\right] \\
& =\mathbf{A d}^{-1} \boldsymbol{K}_{\mathcal{B}} \mathbf{A d}^{-T} \boldsymbol{\Delta} \boldsymbol{X}_{A}
\end{aligned}
$$

From this relation, the conversion rule for stiffness matrices can be identified as, [23]

$$
K_{\mathcal{A}}=\mathbf{A d}^{-1} \boldsymbol{K}_{\mathcal{B}} \mathbf{A d}^{-\mathrm{T}}
$$

It is now possible to derive the symbolic compliance matrix $\boldsymbol{K}_{\text {plat }}$ of the proposed structure. This matrix will be constructed, based on the individual stiffness matrix of one flexible joint $\boldsymbol{K}_{\text {beam }}$, derived in SECTION III.

The proposed platform design, as illustrated in Figure 1, is composed of three flexible joints that form a parallel chain of connections. For a parallel topology, the overall stiffness is obtained by summing up all the individual stiffness matrices:

$$
K_{\text {plat }}=\sum_{i=1}^{3} \operatorname{Ad}_{i}^{-1} K_{\text {beam }} \operatorname{Ad}_{i}^{-\mathrm{T}} .
$$

The wrench transformation matrix $\mathbf{A d}_{i}$ used in this equation represents the transformation from the local frame of flexible joint $i$ to the global reference frame. Hereby, we can define the local coordinate frame of each joint as beeing located at the free end of the beam element (i.e. node B in Figure 2). The beams are arranged in a radial pattern around the platform centre, equally separated by angles of $120^{\circ}$. As a consequence, the local $x$-axis always points towards the platform centre and the local $y$-axis is parallel to the global $y$-axis for each beam. This means that the global frame is obtained by translating the local frame along the local $x$-axis by a distance $r$ (i.e. the radius of the inner plate), followed by a rotation around the local $y$-axis of $0^{\circ}, 120^{\circ}$ or $240^{\circ}$, for each of the three beams respectively. Therefore, the three expressions of $\mathbf{A d}_{i}$ can be expressed using the definitions (16) and (17),

$$
\mathbf{A d}_{\mathrm{i}}=\left[\begin{array}{cc}
\boldsymbol{R}_{i} & \mathbf{0} \\
\boldsymbol{D}_{i} \boldsymbol{R}_{i} & \boldsymbol{R}_{i}
\end{array}\right]
$$

where

$$
\boldsymbol{R}_{i}=\boldsymbol{R}_{y}\left(\frac{2 \pi}{3}(i-1)\right)=, \boldsymbol{D}_{i}=\left[\begin{array}{ccc}
0 & 0 & 0 \\
0 & 0 & r \\
0 & -r & 0
\end{array}\right] .
$$

The matrix $\boldsymbol{R}_{y}$ represents the rotation about the $y$-axis of an angle $\alpha$ in radians,

$$
\boldsymbol{R}_{y}(\alpha)=\left[\begin{array}{ccc}
\cos (\alpha) & 0 & \sin (\alpha) \\
0 & 0 & 0 \\
-\sin (\alpha) & 0 & \cos (\alpha)
\end{array}\right]
$$

By inserting equations (21) into equation (20), the stiffness matrix $\boldsymbol{K}_{\text {plat }}$ of the platform is obtained. The corresponding compliance matrix, on the other hand, can be obtained via the inverse of the stiffness matrix, $\boldsymbol{C}_{\text {plat }}=\boldsymbol{K}_{\text {plat }}{ }^{-1}$.

Due to the symmetrical arrangement of the three flexible joints, both of these matrices are rotationally invariant. Therefore, the overall stiffness and compliance matrices obtained through this method are diagonal matrices:

$$
\begin{aligned}
\boldsymbol{C}_{\text {plat }} & =\operatorname{diag}\left[\begin{array}{llllll}
C_{11} & C_{22} & C_{33} & C_{44} & C_{55} & C_{66}
\end{array}\right], \\
\boldsymbol{K}_{\text {plat }} & =\operatorname{diag}\left[\begin{array}{llllll}
K_{11} & K_{22} & K_{33} & K_{44} & K_{55} & K_{66}
\end{array}\right] .
\end{aligned}
$$

This means that all displacement components are uncorrelated with one another. Furthermore, each displacement component is directly proportional to one force component. For instance, applying a force to the platform that is pointing in $x$-direction will only produce a displacement in the $x$-direction. Likewise, the torque component $T_{x}$ will only affect the angle $\theta_{x}$, etc.

Finally, we can insert the compliance matrix for a cantilever beam seen in equation (13) into equation (20), by setting $\boldsymbol{K}_{\text {beam }}=\boldsymbol{K}_{\text {TIM }}$. This yields the symbolic compliance matrix of the entire platform:

$$
\begin{gathered}
C_{11}=\frac{2\left(1+\Phi_{y}\right) L^{3}}{\left(1+\Phi_{y}\right) 3 A E L^{2}+36 E I_{y}}, \\
C_{22}=\frac{\left(1+\Phi_{z}\right) L^{3}}{36 E I_{z}}, \\
C_{33}=C_{11}, \\
C_{44}=\frac{2 L^{3}}{3\left(\left(4+\Phi_{z}\right) E I_{z} L^{2}+\left(1+\Phi_{z}\right) G J L^{2}+12 E I_{z} r^{2}+12 E I_{z} L r\right)}, \\
C_{55}=\frac{\left(1+\Phi_{y}\right) L^{3}}{3 E I_{y}\left(\left(4+\Phi_{y}\right) L^{2}+12 L r+12 r^{2}\right)}, \\
C_{66}=C_{44} .
\end{gathered}
$$

Note that the corresponding stiffness matrix components $K_{i i}$ are equal to the inverses of these expressions. 


\section{FORCE SENSING}

6-DoF force sensing is essential for understanding the interactions between the ultrasound probe and the human body (e.g. sliding friction [24] and centroid of the applied pressure [25]), as well as for achieving adaptive interaction control [26]. Force sensing can be achieved by measuring the displacement of the inner probe holder. Multiplying these displacement measurements with the stiffness matrix from SECTION IV yields the applied force. In order to estimate the accuracy of this method, FEM simulations were carried out in SolidWorks and directly compared to the analytical model.

\section{A. Simulation Setup}

Figure 3 shows the mesh that was used for this analysis. For the rigid components, a relatively large element size of $7.88 \mathrm{~mm}$ was chosen. For the flexible joints, a finer mesh was used, with an element size of $2.11 \mathrm{~mm}$. During the tests, a force was simulated at the platform centre and the resulting displacement of the platform was recorded. In order to obtain comparable results to the analytical model, the forces had to be exerted precisely at the platform centre, located within the body. For this reason, a small spherical hole was extruded from the centre of the platform. During the simulation, all forces and moments where applied on the surface of this sphere. As the sphere's diameter of $1 \mathrm{~cm}$ is relatively small compared to the dimensions of the platform, it can be considered as a point contact. The peak forces and torques exerted during ultrasound examinations were assumed as $F_{x}=F_{z}=10 \mathrm{~N}, F_{y}=20 \mathrm{~N}, T_{x}=T_{z}=0.5 \mathrm{Nm}, T_{y}=0.2 \mathrm{Nm}$, based on the measurements described in [27].

\section{B. Simulation Results}

The described simulations were carried out for each axis separately, with forces and moments of different magnitudes within the defined application range. The results of these measurements are given in Figure 4. The black dashed line represents the values obtained through FEA. For comparison, the models of the Euler-Bernoulli and Timoshenko beam theories are plotted in red and blue respectively. As predicted by the analytical models, the measurements in the $z$-axis produced the same values as for the $x$-axis (recall that the $x z$ plane is the horizontal plane). In the FEA simulation, the values for these two axes differed by less than $1 \%$. For this reason, the results of the $z$-axis are not shown. Figure 4 only displays the $x$-axis and $y$-axis, hereafter denoted as horizontal axis and vertical axis respectively.

The first graph visualises the compliance in horizontal directions (i.e. the $x z$-plane). It shows that both the EulerBernoulli model and the Timoshenko model deviate from the FEA tests by approximately $+5 \%$. This is the only degree of freedom, where there is no significant difference between the Euler-Bernoulli and Timoshenko beam bending models. The second and the third graph are the most relevant to the manipulation of an ultrasound probe, since they represent forces in the vertical axis and torques about a horizontal axis. In these cases, the Timoshenko model is much closer to the FEA results, with a deviation of roughly $+9 \%$. The EulerBernoulli model on the other hand underestimates the compliance, with a deviation of approximately $-27 \%$. The last graph represents twists around the vertical axis. Both the Timoshenko and Euler-Bernoulli models overestimate the

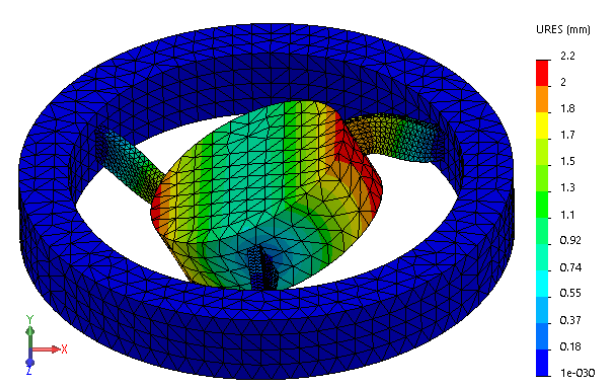

Figure 3. Compliance Simulation using FEA (condition: $T_{z}=0.5 \mathrm{Nm}$ )
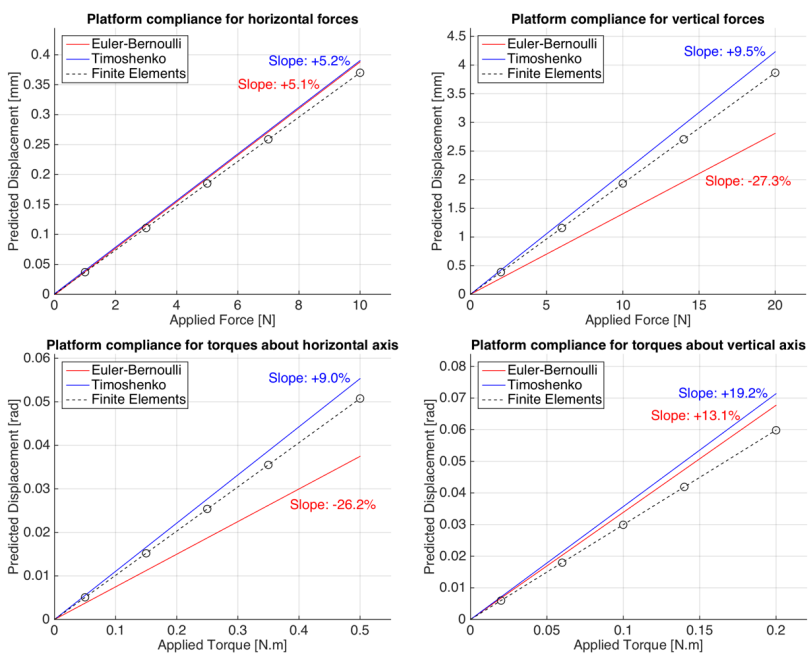

Figure 4. Compliance obtained through Timoshenko model, EulerBernoulli model and FEA

compliance for this case, with $+19 \%$ and $+13 \%$ deviation respectively.

Force sensing based on displacement measurements is the reciprocal case of the above measurements. When the displacement of the platform centre is measured, it can be converted into a force, using the stiffness matrix, which is the inverse of the compliance matrix (i.e. the inverse of the slopes of the graphs in Figure 4). The data obtained through the FEA measurements reflects therefore how accurate force sensing will turn out, when using the Euler-Bernoulli model and the Timoshenko model.

Table 1 shows how much force measurements based on the analytical models will deviate from the FEA simulation. In general, the Timoshenko model produces more reliable results than the Euler-Bernoulli model. The average absolute error of the former is $8.54 \%$ with a standard deviation of $4.09 \%$, whereas the latter produces an average absolute error of $21 \%$ with a standard deviation of $23.85 \%$. Furthermore, it can be stated that the Timoshenko model has the tendency to overestimate the compliance for all degrees of freedom. This systematic error indicates the presence of some specific mechanism that is not covered by the Timoshenko theory (and hence neither by the more approximate Euler-Bernoulli theory). 
TABLE I. ACCURACY OF FORCE SENSING

\begin{tabular}{|c|c|c|c|}
\hline Model & Displacement & $\begin{array}{c}\text { Compliance } \\
{\left[\mathrm{N}^{-1} \mathrm{~m}\right] \text { or }\left[\mathrm{rad} \mathrm{N}^{-1} \mathrm{~m}^{-1}\right]}\end{array}$ & $\begin{array}{c}\text { Equivalent } \\
\text { Error for Force } \\
\text { Measurements }\end{array}$ \\
\hline \multirow{4}{*}{$\begin{array}{l}\text { Finite } \\
\text { Element }\end{array}$} & $\mathrm{X}, \mathrm{Z}$ - linear & $3.700 \mathrm{E}-5$ & - \\
\hline & $\mathrm{Y}$ - linear & $1.933 \mathrm{E}-4$ & - \\
\hline & $\mathrm{X}, \mathrm{Z}-$ angular & $1.015 \mathrm{E}-1$ & - \\
\hline & $\mathrm{Y}$ - angular & $2.994 \mathrm{E}-1$ & - \\
\hline \multirow{4}{*}{ Timoshenko } & $\mathrm{X}, \mathrm{Z}$ - linear & $3.893 \mathrm{E}-5$ & $-4.96 \%$ \\
\hline & $\mathrm{Y}$ - linear & $2.116 \mathrm{E}-4$ & $-8.64 \%$ \\
\hline & $\mathrm{X}, \mathrm{Z}-$ angular & $1.106 \mathrm{E}-1$ & $-8.27 \%$ \\
\hline & $\mathrm{Y}$ - angular & $3.570 \mathrm{E}-1$ & $-16.13 \%$ \\
\hline \multirow{4}{*}{$\begin{array}{l}\text { Euler- } \\
\text { Bernoulli }\end{array}$} & $\mathrm{X}, \mathrm{Z}$ - linear & $3.890 \mathrm{E}-5$ & $-4.88 \%$ \\
\hline & $\mathrm{Y}$ - linear & $1.405 \mathrm{E}-4$ & $+37.61 \%$ \\
\hline & $\mathrm{X}, \mathrm{Z}-$ angular & $7.490 \mathrm{E}-1$ & $+35.45 \%$ \\
\hline & $\mathrm{Y}$ - angular & $3.386 \mathrm{E}-1$ & $-11.59 \%$ \\
\hline
\end{tabular}

\section{CONCLUSION AND DISCUSSION}

The presented platform is comprised of a movable inner planar disk, surrounded by a fixed circular frame. Rubber joints make the system compliant to exterior forces. FEM simulations showed that the Timoshenko model significantly provides higher accuracy. When performing force sensing based on displacement measurements, the analytical method deviates in average by $-9 \%$ from the FEM model, for all 6 degrees of freedom. The most important degrees of freedom for ultrasound probe manipulation are vertical translations (along the $y$-axis) and tilts of the horizontal plane (around the $x$ and $z$-axis). The corresponding force and torque measurements for these axes had a deviation of $-8.64 \%$ and $-8.27 \%$ respectively, compared to the FEM model. For horizontal translations (along the $x$ and $z$-axis), the difference is slightly smaller. However, for twists about the vertical axis (around the $y$-axis), the measured deviation of $-16.13 \%$ was significantly higher. The proposed force sensing method relies on tracking the displacement and rotation of the inner planar disk. To realize this in practice, we plan to implement a fiber optic displacement sensing approach $[28,29]$ in the future.

\section{REFERENCES}

[1] R. Cobbold, Foundations of Biomedical Ultrasound. Oxford University Press, 2007.

[2] K. Ito, K. Tsuruta, S. Sugano, and H. Iwata, "Evaluation of a wearable tele-echography robot system: FASTele in a vehicle using a mobile network," in Proc. Annu. Int. Conf. IEEE Eng. Med. Biol. Soc. EMBS, pp. 2093-2096, 2011.

[3] A. Vilchis-González, J. C. Avila-Vilchis, and A. García-Torres, "TERMI Robot," in Electronics, Robotics and Automotive Mechanics Conference, no. 4, pp. 464-469, 2007.

[4] D. Stoianovici, C. Kim, F. Schäfer, C. M. Huang, Y. Zuo, D. Petrisor, and M. Han, "Endocavity ultrasound probe manipulators," in IEEE/ASME Trans. Mechatronics, vol. 18, no. 3, pp. 914-921, 2013.

[5] A. Krupa, D. Folio, C. Novales, P. Vieyres, and T. Li, "Robotized Tele-Echography: An Assisting Visibility Tool to Support Expert Diagnostic," in IEEE Syst. J., pp. 1-10, 2014.

[6] L. Nouaille, N. Smith-Guérin, G. Poisson, and P. Arbeille, "Optimization of a 4 dof tele-echography robot," in IEEE/RSJ 2010 Int. Conf. Intell. Robot. Syst. IROS 2010 - Conf. Proc., pp. 3501-3506, 2010.

[7] J. Village and C. Trask, "Ergonomic analysis of postural and muscular loads to diagnostic sonographers," in Int. J. Ind. Ergon., vol. 37, no. 9-10, pp. 781-789, 2007.

[8] A. S. Bin Mustafa, T. Ishii, Y. Matsunaga, R. Nakadate, H. Ishii, K. Ogawa, A. Saito, M. Sugawara, K. Niki, and A. Takanishi, "Development of robotic system for autonomous liver screening using ultrasound scanning device," in 2013 IEEE Int. Conf. Robot. Biomimetics, ROBIO 2013, pp. 804-809, 2013.

[9] H. T. Sen, M. a L. Bell, I. Iordachita, J. Wong, and P. Kazanzides, “A cooperatively controlled robot for ultrasound monitoring of radiation therapy," in IEEE Int. Conf. Intell. Robot. Syst., pp. 3071-3076, 2013.

[10] R. Nakadate, H. Uda, H. Hirano, J. Solis, A. Takanishi, E. Minagawa, M. Sugawara, and K. Niki, "Development of assisted-robotic system designed to measure the wave intensity with an ultrasonic diagnostic device," in 2009 IEEE/RSJ Int. Conf. Intell. Robot. Syst. IROS 2009, pp. 510-515, 2009.

[11] Y. Noh, R.J. Housden, A. Gomez, C. Knight, F. Garcia, H. Liu, R. Razavi, K. Rhode, K. Althoefer, "An Ergonomic Handheld Ultrasound Probe providing Contact Forces and Pose Information," in 37th Annu. Int. Conf. IEEE Eng. Med. Biol. Soc. EMBC'15, pp 5773-5776, 2015.

[12] N. Koizumi, S. Warisawa, M. Nagoshi, H. Hashizume, and M. Mitsuishi, "Construction Methodology for a Remote Ultrasound Diagnostic System," in Robot. IEEE Trans., vol. 25, no. 3, pp. 522$538,2009$.

[13] K. Ito, S. Sugano, and H. Iwata, "Portable and attachable teleechography robot system: FASTele," in 2010 Annu. Int. Conf. IEEE Eng. Med.Biol. Soc. EMBC'10, pp. 487-490, 2010.

[14] R. Nakadate, Y. Tokunaga, J. Solis, A. Takanishi, E. Minagawa, M. Sugawara, K. Niki, and A. Saito, "Development of robot assisted measurement system for abdominal ultrasound diagnosis," in $20103 \mathrm{rd}$ IEEE RAS EMBS Int. Conf. Biomed. Robot. Biomechatronics, BioRob 2010, pp. 367-372, 2010.

[15] L. P. Jentoft, A. M. Dollar, C. R. Wagner, and R. D. Howe, "Intrinsic embedded sensors for polymeric mechatronics: Flexure and force sensing," in Sensors(Switzerland), vol. 14, no. 3, pp.3861-3870, 2014.

[16] C. Qiu, P. Qi, H. Liu, K. Althoefer, J.S. Dai, "Six-Dimensional Compliance Analysis and Validation of Ortho-Planar Springs," in Journal of Mechanical Design, 138(4), 042301, 2016.

[17] P. Qi, C. Qiu, H. Liu, J.S. Dai, L. Seneviratne, K. Althoefer, "A Novel Continuum Manipulator Design using Serially Connected DoubleLayer Planar Springs," in IEEE/ASME Trans. Mechatronics, 2016, in press.

[18] T. Noh, "Development of a mechanically coupled, 6-axis ForceTorque sensor for Humanoid robots," M.S. thesis, Korea Advanced Institute of Science and Technology, 2006.

[19] L. Andersen and S. Nielsen, "Elastic Beams in Three Dimensions," in Structural Mechanics, DCE Lecture Notes No. 23, Aalborg University, 2008.

[20] H. P. Gavin, "Structural Element Stiffness, Mass, and Damping Matrices," in CEE 541. Structural Dynamics, Duke University, 2014.

[21] J.S. Dai, "Finite displacement screw operators with embedded Chasles' motion," in Journal of Mechanisms and Robotics, Trans. ASME, 4(4): 041002, 2012.

[22] R. M. Murray, Z. Li, and S. S. Sastry, A Mathematical Introduction to Robotic Manipulation, vol. 29. 1994.

[23] J.S. Dai, X.L. Ding, "Compliance analysis of a three-legged rigidlyconnected platform device," in Journal of Mechanical Design, Trans. ASME, 128(4): 755-764, 2006.

[24] X. Song, H. Liu, K. Althoefer, T. Nanayakkara, L.D. Seneviratne, "Efficient Break-Away Friction Ratio and Slip Prediction Based on Haptic Surface Exploration," in IEEE Transactions on Robotics, $30(1), 203-219,2014$

[25] H. Liu, K.C. Nguyen, et al., "Finger contact sensing and the application in dexterous hand manipulation," in Autonomous Robots, 39(1), 25-41, 2015.

[26] J. Back, J. Bimbo, Y. Noh, L. Seneviratne, K. Althoefer, H. Liu, "Control a contact sensing finger for surface haptic exploration," in IEEE Int. Conf. Robot Autom (ICRA), 2736-2741, 2014.

[27] M. W. Gilbertson and B. W. Anthony, "An ergonomic, instrumented ultrasound probe for 6-axis force/torque measurement," in Proc. Annu. Int. Conf. IEEE Eng. Med. Biol. Soc. EMBS, pp. 140-143, 2013.

[28] T.C. Searle, K. Althoefer, L. Seneviratne, H. Liu, "An optical curvature sensor for flexible manipulators," in IEEE Int. Conf. Robot Autom (ICRA), 4415-4420, 2013.

[29] H. Liu, J. Li, X. Song, L. Seneviratne, K. Althoefer, "Rolling Indentation Probe for Tissue Abnormality Identification During Minimally Invasive Surgery", in IEEE Transactions on Robotics, $27(3), 450-460,2011$ 